\title{
Quark model description of baryon properties
}

\author{
Bernard Ch. Metsch ${ }^{1, \star}$ and Herbert-R. Petry \\ ${ }^{1}$ Helmholtz Institut für Strahlen- und Kernphysik, Nußallee 14-16, D 53115 Bonn, Germany
}

\begin{abstract}
The goal of this project was a systematic calculation, consistent with the mass spectrum, of electroweak properties of baryons, as well as of the hadronic two-body decays of baryon resonances. The basis of the calculations was a relativistically covariant quark model for mesons and baryons, which allows for the calculation of (momentum dependent) hadronic form factors and coupling vertices in the relevant photon and hadron induced reactions and simultaneously describes the hadronic mass spectrum up to $3 \mathrm{GeV}$.
\end{abstract}

\section{Introduction}

We developed a constituent quark model for baryons and mesons within a field-theoretical framework starting from the homogeneous Bethe-Salpeter for bound hadronic states. This model thus fully respects Poincaré covariance, mandatory to describe deeply bound states and/or scattering processes at larger momentum transfer.

Before the start of the first funding period of the SFB/TR16 this model, which describes the quark dynamics through an instantaneous confinement potential with a suitable Dirac structure, rising linearly with interquark distances and a spin-flavour dependent interaction motivated by instanton effects to describe the major spin-dependent mass splittings, had been applied to a calculation of the spectrum of light flavoured mesons and baryons up to masses of about $3 \mathrm{GeV}$, see e.g. [1-3] as well as some selected electroweak observables such as electromagnetic form factors, charge radii and magnetic moments, see e.g. $[4,5]$.

\section{Earlier achievements}

During the first funding period the following issues have been investigated, also see [6]:

\section{Electromagnetic properties:}

The calculation of some momentum transfer dependent helicity amplitudes as they occur in electro- or photoinduced reactions on the basis of the Salpeter amplitudes determined by the mass spectrum is (in lowest order in the Mandelstam formalism) parameter free. These have been calculated systematically both for non-strange baryons [7] and for hyperons, see [8]. For non-strange photo-excitation it was found, that sizeable transition amplitudes are to be expected only for the lowest states of each total spin. Higher excited states can therefore not

\footnotetext{
^e-mail: metsch@hiskp.uni-bonn.de
}

be directly reached in photo-production experiments. To exemplify the quality of the prediction a comparison of calculated and experimental helicity amplitudes at a finite momentum transfer of $Q^{2}=0.65 \mathrm{GeV}^{2}$ is shown in Table 1 .

Table 1. Calculated helicity amplitudes (in units of $10^{-3} \mathrm{GeV}^{-\frac{1}{2}}$ ) of proton resonances at $Q^{2}=0.65 \mathrm{GeV}^{2}$ compared to data from a combined analysis of single- and double-pion electroproduction.

\begin{tabular}{crrrcccc}
\hline & \multicolumn{3}{c}{ Calc. } & & \multicolumn{3}{c}{ Aznauryan et al.[9] } \\
\cline { 2 - 3 }$B^{*}$ & $A_{p}^{1 / 2}$ & $A_{p}^{3 / 2}$ & $S_{p}^{1 / 2}$ & & $A_{p}^{1 / 2}$ & $A_{p}^{3 / 2}$ & $S_{p}^{1 / 2}$ \\
\hline$P_{11}(1440)$ & 62 & & 17 & $21 \pm 4$ & & $33 \pm 6$ \\
$D_{13}(1520)$ & -55 & 19 & -20 & $-65 \pm 4$ & $62 \pm 5$ & $-35 \pm 4$ \\
$S_{31}(1620)$ & 2 & & -14 & $16 \pm 4$ & & $-28 \pm 3$ \\
$S_{11}(1650)$ & 1 & & -3 & $43 \pm 7$ & & $-6 \pm 3$ \\
$F_{15}(1680)$ & -51 & 25 & -28 & $-32 \pm 5$ & $51 \pm 4$ & $-15 \pm 3$ \\
$D_{33}(1700)$ & 49 & 41 & -15 & $44 \pm 4$ & $36 \pm 4$ & $-7 \pm 4$ \\
$D_{13}(1700)$ & -19 & -7 & -6 & $-21 \pm 2$ & $10 \pm 1$ & 0 \\
$P_{13}(1720)$ & 66 & -16 & -10 & $55 \pm 3$ & $-68 \pm 4$ & 0 \\
\hline & & & & & & & \\
\hline
\end{tabular}

Although helicity amplitudes calculated for hyperons are not directly accessible in experiments, they play a key role in some contributions to strangeness photo- and electroproduction. As for the non-strange baryons it was found that many amplitudes are larger in magnitude at a finite momentum transfer than at the photon point. It was concluded in [8] that interesting isospin dependencies occur when comparing the decay of hyperons in $\Lambda$ - with those in $\Sigma$-resonances. Furthermore the calculated photonic decay width for $\Lambda(1405) \rightarrow \Lambda(1116)+\gamma$ exceeds the experimental value by at least an order of magnitude and thus lends further support to the conclusion that $\Lambda(1405)$ can not merely be described as a dominant three-quark excited state. In contrast the electromagnetic decay of the lowest negative parity $\Sigma(1620)$ resonance can be well accounted for by the present quark model calculation. 
In a field theoretical context static electromagnetic observables, such as electric charge radii and magnetic moments are usually extracted from elastic form factors, i.e. by extrapolation to vanishing momentum transfer of matrix elements of appropriate current operators, a procedure which partially suffers from numerical inaccuracies. In a novel approach $[10,11]$ developed in the first funding period this circuitous route was circumvented and such observables can, very similar to the procedure in nonrelativistic quantum mechanics, be computed directly (and numerically more accurately) as expectation values of operators with respect to the Salpeter amplitudes obtained from the calculation of the mass spectrum in the baryon rest frame. The expressions, derived essentially from linear response, are readily interpreted physically, possess an obvious non-relativistic limit and allow for a discrimination of e.g. the spin- and orbital angular momentum contribution to the magnetic moments. Some results are compared to experimental data in Table 2, where also the re-

Table 2. Comparison of calculated (BS) and experimental magnetic moments (in units of $\mu_{N}$ ) of some selected baryon resonances. Experimental data from [12] and [a] [13], [b] [14], [c] [15]. The column labeled PFSA contains the results obtained in [16].

\begin{tabular}{crrr}
\hline $\mathrm{B}$ & $\mathrm{BS}$ & \multicolumn{1}{c}{ Exp. } & PFSA \\
\hline $\mathrm{p}$ & 2.77 & 2.793 & 2.70 \\
$\mathrm{n}$ & -1.71 & -1.913 & -1.70 \\
$\Lambda$ & -0.61 & $-0.613 \pm 0.004$ & -0.65 \\
$\Sigma^{+}$ & 2.51 & $2.458 \pm 0.01$ & 2.35 \\
$\Sigma^{0}$ & 0.75 & - & 0.72 \\
$\Sigma^{-}$ & -1.02 & $-1.16 \pm 0.025$ & -0.92 \\
$\Xi^{0}$ & -1.33 & $-1.25 \pm 0.014$ & -1.24 \\
$\Xi^{-}$ & -0.56 & $-0.6507 \pm 0.0025$ & -0.68 \\
$\Delta^{++}$ & 4.14 & $3.7-7.5$ & 4.17 \\
$\Delta^{+}$ & 2.07 & $2.7^{+1.0} \pm 1.5 \pm 3^{[a]}$ & 2.08 \\
$\Delta^{0}$ & 0 & - & 0 \\
$\Delta^{-}$ & -2.07 & - & -2.08 \\
$\Sigma^{*+}$ & 2.51 & - & 2.07 \\
$\Sigma^{* 0}$ & 0.27 & - & 0.09 \\
$\Sigma^{*-}$ & -1.97 & - & -1.89 \\
$\Xi^{* 0}$ & 0.59 & - & 0.18 \\
$\Xi^{*-}$ & -1.83 & - & -1.73 \\
$\Omega^{-}$ & -1.66 & $2.02 \pm 0.05^{[b, c]}$ & -1.59 \\
$\mathrm{P}^{11}(1440)^{+}$ & 1.55 & - & - \\
$\mathrm{D}^{13}(1520)^{+}$ & 1.44 & - & - \\
$\mathrm{S}^{11}(1535)^{+}$ & 0.37 & - & - \\
$\mathrm{S}^{11}(1650)^{+}$ & 1.85 & - & - \\
\hline & & &
\end{tabular}

sults obtained by the Graz group [16], which use Dirac's point form formulation of relativistic quantum mechanics to calculate the relevant current matrix elements. Although at first face this is a completely different method the results are strikingly similar; in both methods the importance of relativistic covariance is paramount. The new method also allows for a prediction of magnetic moments, see also Table 2, of excited baryons, a research topic of some interest within the SFB/TR16, also see project B.4.

\section{Baryonic semi-leptonic decays:}

As an additional (again parameter free) application and therefore genuine test of our relativistic quark model, we calculated weak decays of the ground state decuplet and octet states with light flavours and also the semi-leptonic decay of e.g. $\Lambda_{c}$, see also below. The computed observables $[17,18]$ include helicity amplitudes, axial-vector to vector coupling ratios, weak-magnetism ratios, differential and total decay rates of electronic and muonic semileptonic baryon decays. We have compared our theoretical predictions with all available experimental data and in general found a good agreement. We have given many predictions, as an example see Fig. 1, of observables for which

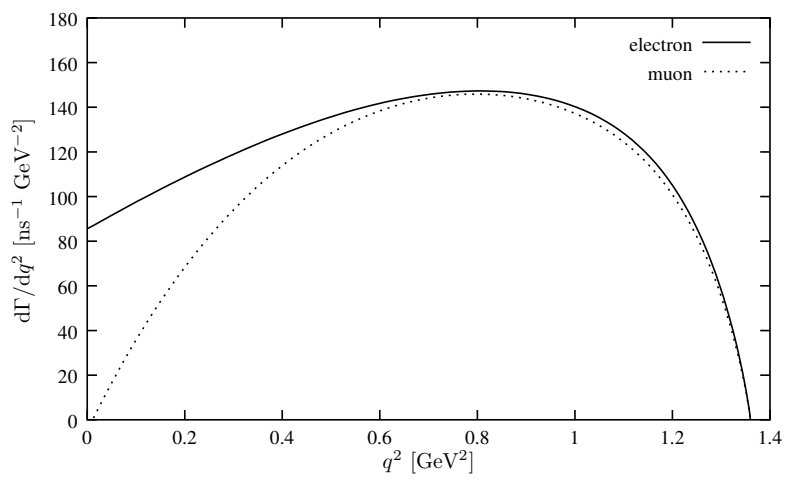

Figure 1. Calculated differential decay width for the semileptonic decay $\Lambda_{c}^{+} \rightarrow \Lambda^{0} \ell^{+} \bar{v}_{\ell}$, for $\ell=e$ (solid line) and $\ell=\mu$ (dotted line).

currently no experimental results exist. There clearly exists an experimental deficit regarding differential decay rates of semi-leptonic baryon decays. An experimental verification of the predicted axial-vector to vector coupling ratio $g_{A} / g_{V}=-0.95$ for the decay $\Lambda_{c}^{+} \rightarrow \Lambda^{0} e^{+} \bar{v}_{e}$ would be very important in this respect.

\section{Strong decays:}

Like the electroweak amplitudes discussed above, strong two-body decays of baryon resonances can in lowest order in the context of the Mandelstam formalism be calculated parameter free. Because of the neglect of important hadronic final state interactions in this approximation, a quantitatively accurate reproduction of experimental decay widths is hardly to be expected, and, in fact, the calculated values are in general too small. Nevertheless, in a systematic theoretical study of roughly 1700 strong twobody decays of light-flavoured baryon excitations into a pseudoscalar octet meson and a (possibly excited) baryon final state [18], as performed during the first funding period, it was found that the theoretically investigated strong decays can be classified into three categories: those (about $15-20 \%$ ) for which the calculated partial decay width is relatively large and numerically a substantial fraction of 


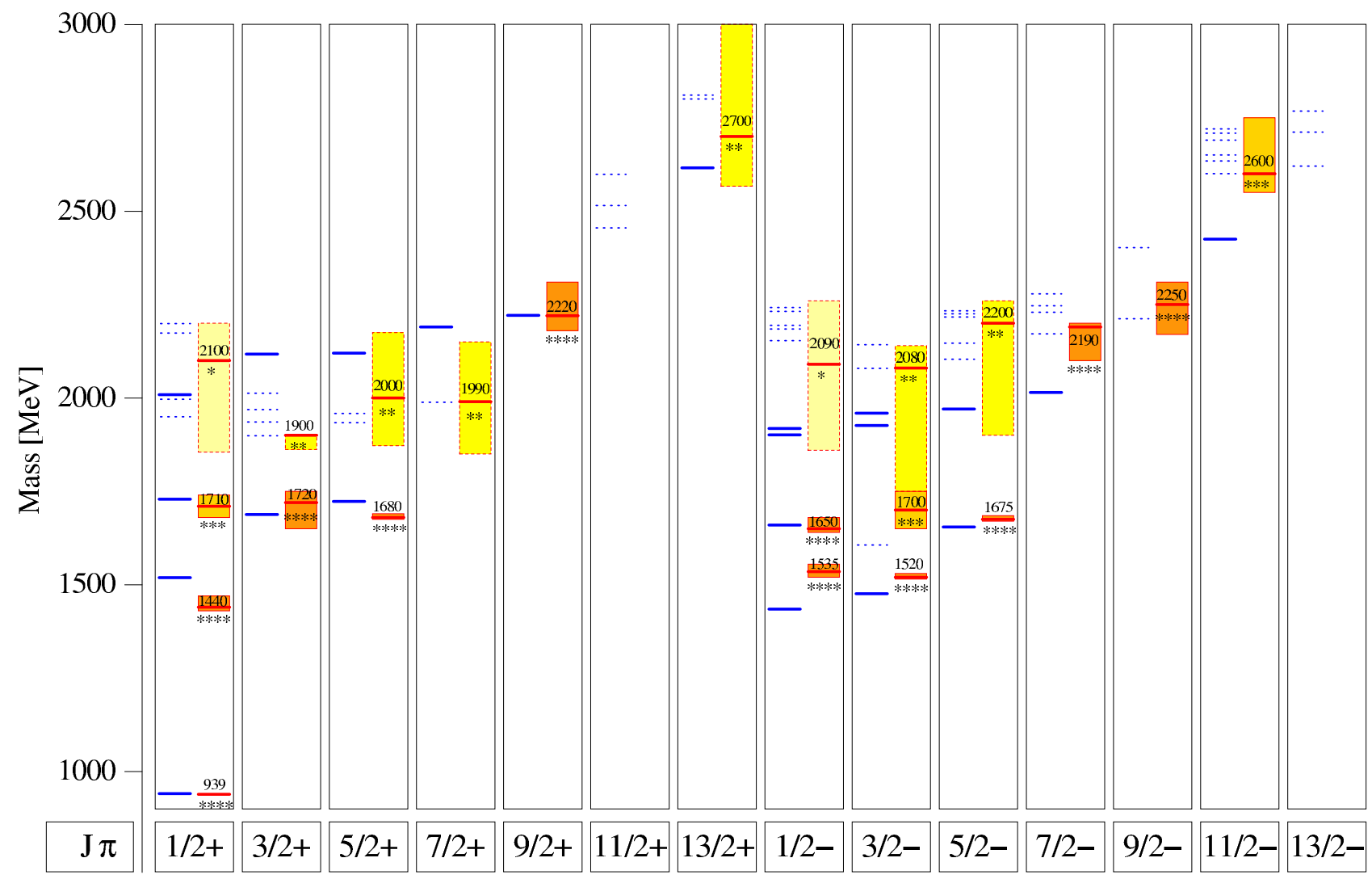

Figure 2. The calculated nucleon resonance spectrum (left in each column) compared to the experimental masses (right in each column) from [12]. Dotted lines indicate that the calculated $\pi N$-decay width of the resonance vanishes, a solid line indicates those states for which the calculated width is finite and for most states at least in qualitative agreement with the experimental value.

the experimental value, those for which the calculated width is small but clearly non-zero and finally those with (virtually) vanishing decay width. The resonances with decays belonging to the first and second categories are indeed those which have been observed experimentally in $\pi$ or $K$-nucleon scattering, see e.g. Fig. 2, explaining why the majority of the excited states could not be detected in such reactions. The results can be summarised as follows:

- The present calculation does offer a solution to the problem of the missing resonances: They simply do not couple to $N \pi$ or $N \bar{K}$.

- Most of the observed resonances indeed have a nonvanishing theoretical coupling to $N \pi$ or $N \bar{K}$.

- The results allow for unique assignments between theoretical and experimental states. These assignments are not possible based on the mass spectra alone.

- For $N$ - and $\Lambda$-resonances $N \pi$ and $N \bar{K}$ are the prefered decay channels. For each set of resonances about $60 \%$ of the couplings vanish and about $25 \%$ calculated decay widths are larger than $5 \mathrm{MeV}$.

- Most $\Delta$ - and $\Sigma$-resonances do not decay into $N \pi$ - or $N \bar{K}$, respectively. For each set of resonances about $80 \%$ of the couplings vanish and about 5\% of the decay widths are larger than $5 \mathrm{MeV}$. For these decuplet state some decays are theoretically prefered: $\Delta \rightarrow \Delta \eta, \Sigma \rightarrow \Sigma \pi$ and $\Sigma \rightarrow \Delta \bar{K}$.
- Clear selection rules with respect to an intrinsic spinflip $\left(\frac{3}{2} \rightarrow \frac{1}{2}\right)$ were observed: Such a spin-flip transition is prefered when $\Lambda$ is a final state, is not relevant when a $\Delta$ is involved somewhere and is avoided in all the other cases.

- In general even the non-vanishing decay widths tend to be too small compared to experiment. Note however that the decay widths for the missing resonances are very often smaller by even several orders of magnitude.

- There is always a resonance observed in experiment, if the theoretical coupling is non-vanishing to $N \pi$ or $N \bar{K}$, respectively.

- In total about $60 \%$ of the couplings to ground states and $90 \%$ of those to excited states vanish, but there are many allowed quasi-two-body decays for almost every N- or $\Delta$-resonance, including the missing resonances, indicating the possibility to detect these states in quasi-twobody decay cascades.

The calculations could be extended to even more missing states and to strong two-body decays involving vector mesons. Instantons also lead to an effective threebody potential, which, because of its colour dependence is spectroscopically irrelevant but in principle leads to a highly selective three-body contribution to strong decays involving (pseudo)scalar mesons, in particular $\eta$ and $\eta^{\prime}$. It indeed proved to have a strong impact on the numerical 
results for particular strong two-body decays of mesons. Unfortunately, the numerical implementation of 't Hooft's three-body interaction for baryonic decays turned out to be numerically much more involved than it is for mesonic decays where the amplitude essentially factorises. This subject was therefore not further pursued.

In view of the results discussed so far we concluded that this relativistic constituent quark model accounts for the majority of the salient features in the baryon spectrum, such as linear Regge trajectories, the major spin dependent mass splittings, in particular the occurrence of spinparity doublets and offers a parameter-free quantitative description of many electroweak observables, such as static moments, helicity amplitudes and semi-leptonic decay observables. Furthermore, as discussed above, the lowest order calculation of strong meson-baryon decay amplitudes, at least qualitatively explains why many excited baryon states have not been observed in meson-baryon scattering experiments so far.

\section{Deficiences and improvements}

Although this in our opinion validated the calculations performed so far, some unresolved issues remained:

1. The quantitative description of strong two-body decay amplitudes is clearly deficient, probably mainly because final state interactions and thus coupled channel effects are completely ignored. An attempt to improve upon this turned out to be extremely difficult. In the instantaneous approximation it is possible to determine the strong two-body decay amplitudes merely on the mass shell. Even with an adequate parametrisation of non-resonant amplitudes as an ingredient in a simplified coupled channel calculation (essentially in a $K$-matrix approach) the resulting decay widths hardly changed[19] .

2. In the mass spectrum of negative parity $\Delta$ resonances there was significant experimental evidence for the existence of three resonances in the mass range $1900-1940 \mathrm{MeV}$, of which the conspicuous low position could not be accounted for by the calculation as described above, see Fig. 3 . Moreover, also the calculated position of the first excited positive parity $\Delta$ resonances was much too high. An improvement of the description was obtained in the manner described in [22].

\subsection{Effects of an additional spin and flavour dependent interaction}

Among the various possibilities we persued the simple Ansatz to phenomenologically introduce an additional spin and flavour dependent interaction, more or less inspired by the results obtained by the Graz-Group with a version of a so-called Goldstone-boson-exhange interaction, see e.g. [21]. Here, we decided to parameterise the new spinflavour-dependent interaction purely phenomenologically

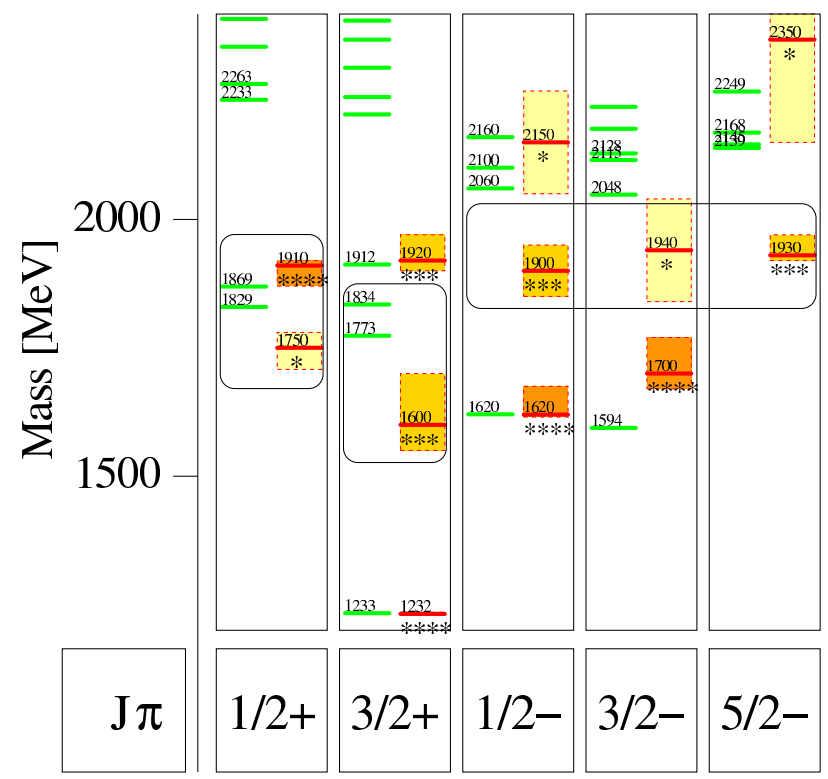

Figure 3. Discrepancies between experimental (right part of each column, from [20]) and calculated (left part of each column, as in [2]) positions of the lowest $\Delta$-resonances. Shaded boxes indicate the uncertainty in the resonance position.

as a local potential in configuration space, its simple form given by

$$
V(\vec{x})=\sum_{a=1}^{8} g_{a}^{2}\left[\lambda^{a} \gamma_{5} \otimes \lambda^{a} \gamma_{5}\right] v_{\lambda_{a}}(|\vec{x}|)
$$

where the Gell-Mann matrices $\lambda^{a}$ determine the flavour-, $\left(\gamma_{5} \cdot \gamma_{5}\right)$ determines the spin- and the simple gaussian

$$
v_{\lambda}(r):=\frac{1}{\lambda^{3} \pi^{\frac{3}{2}}} \exp \left(-\frac{r^{2}}{\lambda^{2}}\right)
$$

determines the interquark-distance- dependence.

After an adjustment of the parameters, for the values, see [22] (and for a slightly modified set of parameter values, see [23]) indeed an improved description of the $\Delta$ mass spectrum was found:, see e.g. Fig. 4, Remarkably enough, in this manner, not only it was possible to account for negative-parity $\Delta$-resonances slightly below $2 \mathrm{GeV}$, but also a much more accurate description of the $\Delta\left(\frac{3}{2}^{+} ; 1660\right)$ and the postive-parity $\Delta\left(\frac{1}{2}^{+}\right)$-resonances was obtained. Furthermore, with this additional spin-flavour dependent interaction the position of the Roper-resonance below the first negative parity nucleon resonances is now accurately accounted for, see Fig. 5 . In addition the masssplittings of these negative-parity resonances are now better reproduced. For similar improvements in the spectra of strange baryons we refer to [22].

\subsection{Impact on electromagnetic observables}

The phenomenological introduction of the additional spinflavour depependent interaction kernel increased the number of parameters of seven in the original model (Model $\mathcal{A}$, 


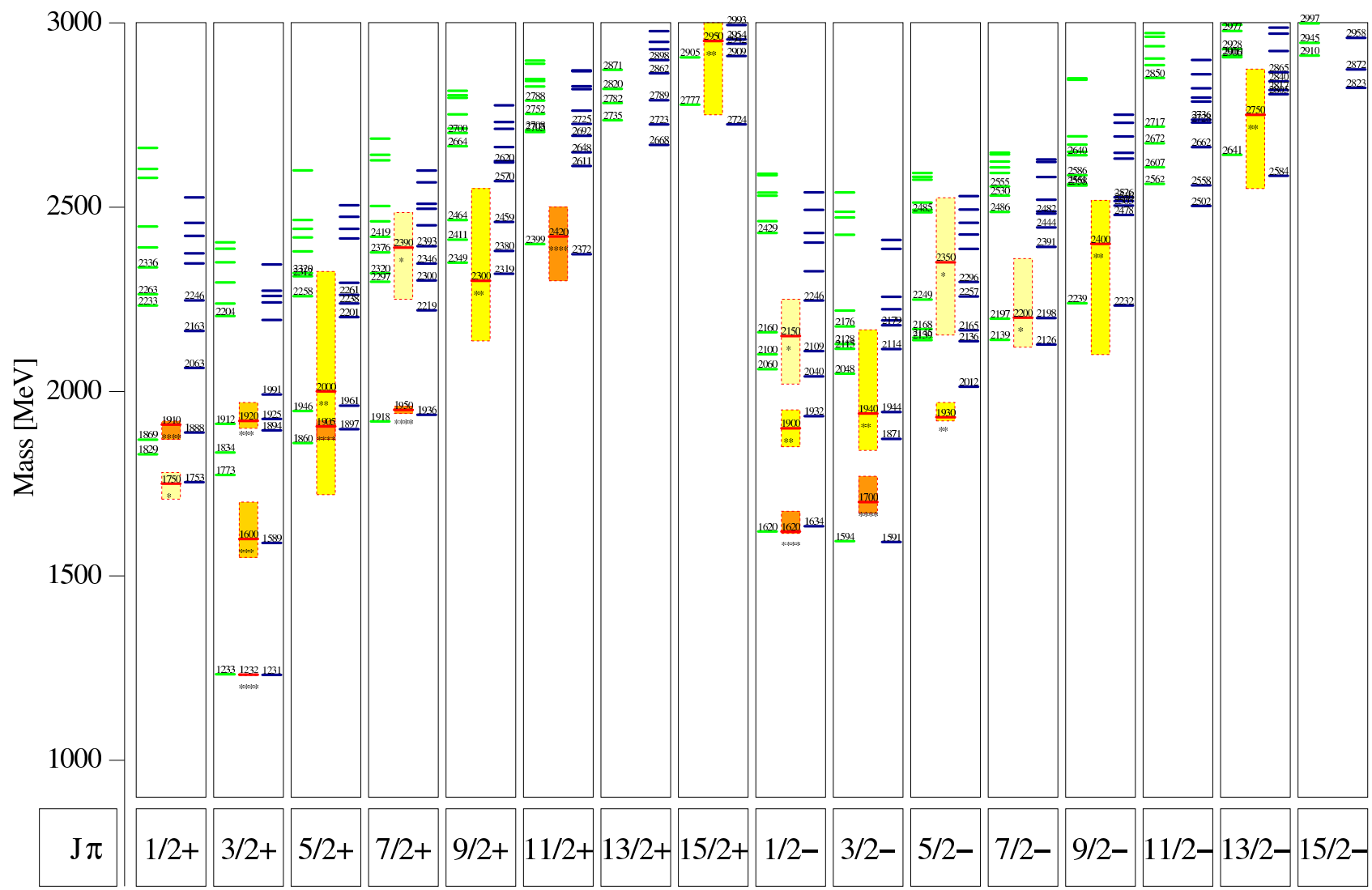

Figure 4. Comparison of the $\Delta$-spectrum calculated with the additional phenomenological spin-flavour dependent interaction (right side of each column), see [22], with experimental data from the Particle Data Group [20] (central in each column) and with the results from the model as discussed in [2] (left side in each column). Shaded boxes indicate the uncertainty in the resonance position.

see $[2,3])$ to ten in the new version (Model $C$, see [23]), which we considered to be still acceptable in view of the multitude of baryon masses described accurately in this manner. As before, after fixing the parameters by a fit to the mass spectra, the calculation of electromagnetic properties in lowest order is a genuine prediction. In Fig. 6 we display the electric form factor of the proton, divided by the dipole-shape

$$
G_{D}\left(Q^{2}\right)=\frac{1}{\left(1+Q^{2} / M_{V}^{2}\right)^{2}},
$$

with $M_{V}^{2}=0.71 \mathrm{GeV}^{2}$ (see [24, 27]) is shown for two versions of parameters (see Table 1 in [23]) in comparison to experimental data.

It is found that with the new model describes the data much better than the original model, see [5]. This applies also to the electric neutron from factor, see Fig. 7; this small quantity is very sensitive to parameter changes and the deviation between both curves could be interpreted as an estimate of the uncertainty in the model prediction. It demonstrates, however, that it is indeed possible to account for the momentum transfer dependence and the position of the maximum rather accurately. Also for the magnetic form factors displayed in Fig. 8 and 9 improvements are observed: this concerns in particular the description of the magnetic proton form factor at higher momentum transfers $Q^{2} \gtrsim 1 \mathrm{GeV}^{2}$. The momentum transfer depen-

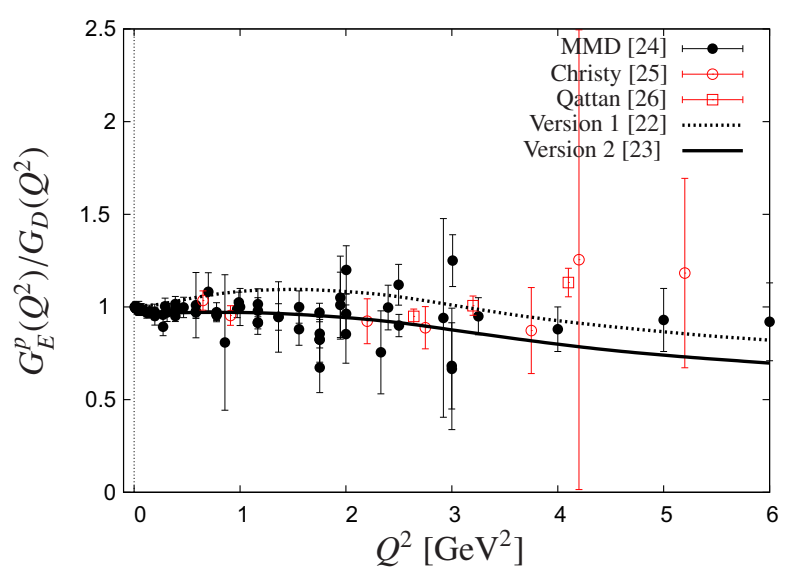

Figure 6. The electric form factor of the proton divided by the dipole form $G_{D}\left(Q^{2}\right)$, Eq. (1). MMD-Data are taken from Mergell et al. [24], supplemented by data from Christy et al. [25] and Qattan et al. [26]. The solid line represents the results with the model parameters listed in [23] while the dashed line those listed in [22]. Red data points are taken from polarisation experiments and black points are obtained by Rosenbluth separation.

dence (up to $Q^{2} \approx 6 \mathrm{GeV}^{2}$ ) of a multitude of transverse and longitudinal helicity amplitudes has been presented and discussed in [23], both in the original model and in 


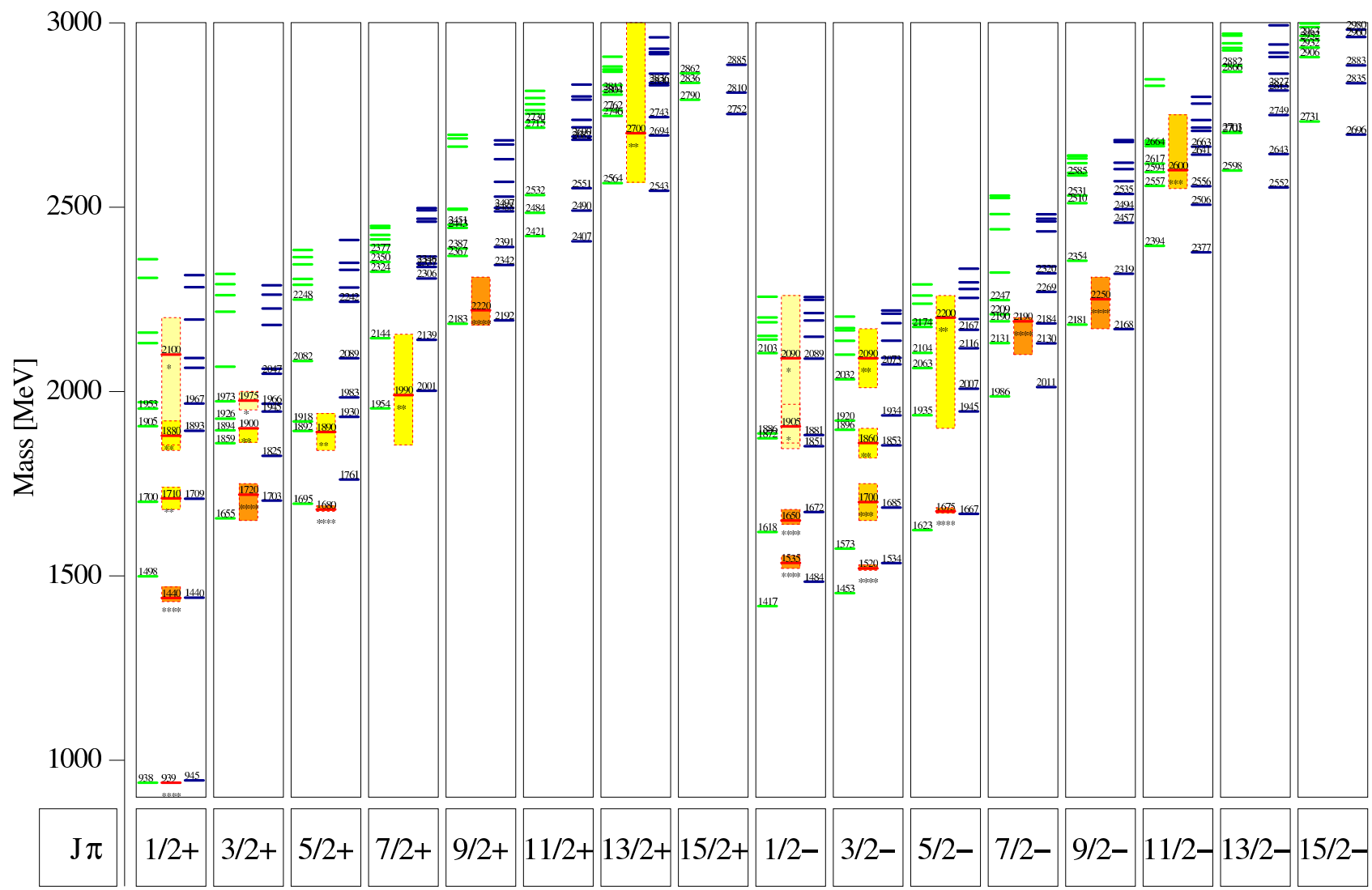

Figure 5. Comparison of the $N$-spectrum calculated with the additional phenomenological spin-flavour dependent interaction (right side of each column), see [22], with experimental data from the Particle Data Group [20] (central in each column) and with the results from the model as discussed in [2] (left side in each column). Shaded boxes indicate the uncertainty in the resonance position.

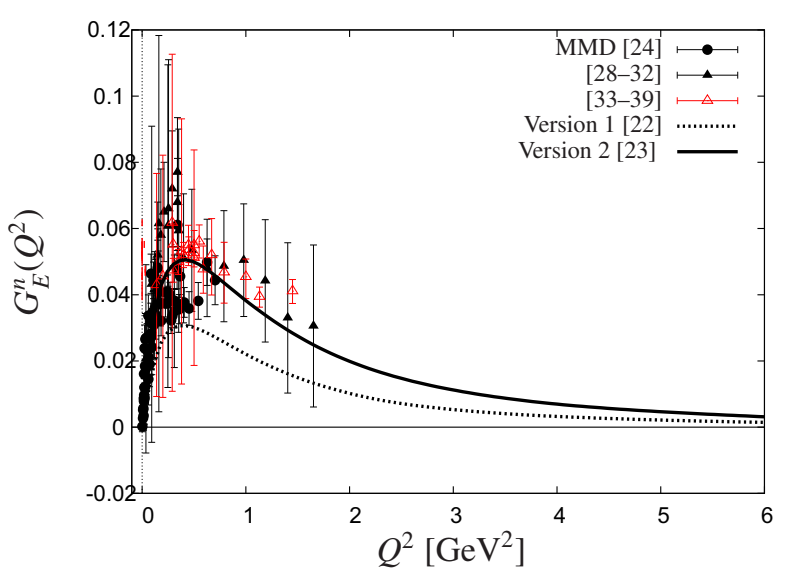

Figure 7. The electric form factor of the neutron. MMD-Data are taken from the compilation of Mergell et al. [24]. The solid line represents the results with the model parameters listed in [23] while the dashed line those listed in [22]. Red data points are taken from polarisation experiments and black points are obtained by Rosenbluth separation.

the model with the additional spin-flavour dependent interaction. Here also results on photocouplings for a few dozen $N \rightarrow N^{*}$ - and $N \rightarrow \Delta^{*}$ - transitions can be found.

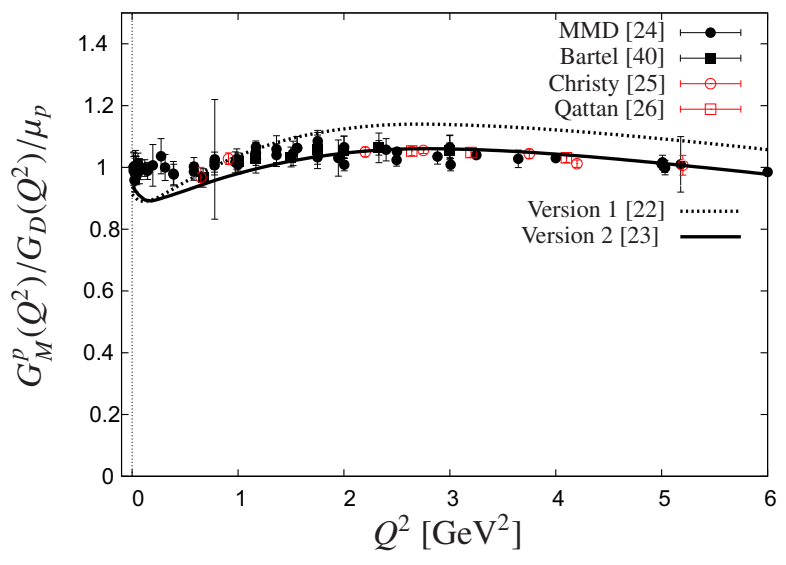

Figure 8. The magnetic form factor of the proton divided by the dipole form $G_{D}\left(Q^{2}\right)$, Eq. (1) and the magnetic moment of the proton $\mu_{p}=2.793 \mu_{N}$. MMD-Data are taken from the compilation of Mergell et al. [24]. Additionally, polarisation experiments are marked in red. The black marked data points are obtained by Rosenbluth separation.

\subsection{Summary}

The same calculational framework as presented by Merten et al. [5] was used for the computation of current-matrix elements on the basis of Salpeter-amplitudes obtained in 


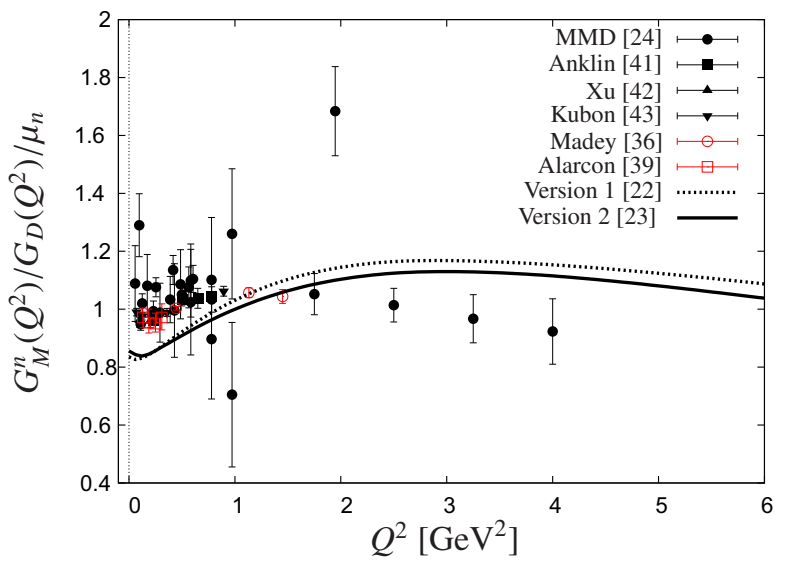

Figure 9. The magnetic form factor of the neutron divided by the dipole form $G_{D}\left(Q^{2}\right)$, Eq. (1) and the experimental value of the magnetic moment of the neutron $\mu_{n}=-1.913 \mu_{N}$. Note that the calcultated value of the neutron magnetic moment is in fact too small, see also Table 2, where it was calulated with an alternative method. MMD-Data are taken from the compilation by Mergell et al. [24] and from more recent results from MAMI [41, 43] . Additionally, polarisation experiments are marked by red data points. The black marked points are obtained by Rosenbluth separation.

a calculation of the baryon mass spectra, see [22], where, in addition to confinement and a spin-flavour dependent interaction motivated by instanton effects as used in an older model version [2, 3], an additional phenomenological short ranged spin-flavour dependent interaction was introduced in order to improve in particular upon the description of some excited negative parity $\Delta$ resonances at $\approx 1.9 \mathrm{GeV}$. In some significant details a superior description of the mass spectrum of all baryon resonances at the expense of only three extra phenomenological parameters was thus obtained. It was found that the description of ground state electromagnetic form factors was also significantly improved. A systematic investigation of electromagnetic transition form factors in the form of momentum transfer dependent transverse and longitudinal helicity amplitudes revealed that both in the original and in the novel model the transverse $A^{\frac{1}{2}}$-helicity amplitudes are significantly better described than the $A^{\frac{3}{2}}$-amplitudes.

Stimulating discussions with E. Klempt, A.V. Sarantsev and U. Thoma within the framework of the DFG supported Collaborative Research Centre SFB/TR16 as well as contributions by Christian Haupt, Sascha Migura, Michael Ronniger and Daniela Tolentino-Zang are gratefully acknowledged.

\section{References}

[1] U. Löring, K. Kretzschmar, B.Ch. Metsch, H.R. Petry, Eur. Phys. J. A 10 (2001) 309-346.

[2] U. Löring, B.Ch. Metsch, H.R. Petry, Eur. Phys. J. A 10 (2001) 395-446.

[3] U. Löring, B.Ch. Metsch, H.R. Petry, Eur. Phys. J. A 10 (2001) 447-486.

[4] U. Löring, K. Kretzschmar, D. Merten, B. Metsch, H.R. Petry, Eur. Phys. J. A 14 (2002) 477-489.
[5] D. Merten, U. Löring, B. Metsch, H.-R. Petry, Eur. Phys. J. A 18 (2003) 193-195.

[6] B.C. Metsch, Eur. Phys. J. A 35 (2008) 275. doi:10.1140/epja/i2007-10557-4.

[7] S. Kreuzer, "Ein massenabhängiges Confinement Potential für das Bethe-Salpeter Modell", Diplomarbeit, Universität Bonn, August 2006;

[8] T. Van Cauteren, J. Ryckebusch, B. Metsch and H. R. Petry, Eur. Phys. J. A 26 (2005) 339. [arXiv:nuclth/0509047].

[9] I. G. Aznauryan, V. D. Burkert, G. V. Fedotov, B. S. Ishkhanov, Phys. Rev. C 72 (2005) 045201d [arXiv:hep-ph/0508057].

[10] C. Haupt, B. Metsch and H. R. Petry, Eur. Phys. J. A 28 (2006) 213 [arXiv:hep-ph/0602151].

[11] C. Haupt, "Electromagnetic properties of Baryons," Dissertation, Universität Bonn, April 2006.

[12] Particle Data Group, W.-M. Yao et al., J. Phys. G 33 (2006) 1 .

[13] M. Kotulla et al., Phys. Rev. Lett. 89 (2002) 272001 [arXiv:nucl-ex/0210040].

[14] N. B. Wallace et al., Phys. Rev. Lett. 74 (1995) 3732.

[15] H. T. Diehl et al., Phys. Rev. Lett. 67 (1991) 804.

[16] K. Berger et al., Phys. Rev. D 70 (2004) 094027 [arXiv:nucl-th/0407009].

[17] S. Migura, D. Merten, B. Metsch and H. R. Petry, Eur. Phys. J. A 28 (2006) 55. [arXiv:hep-ph/0602152]

[18] S. Migura, "Weak and strong baryon decays in a constituent quark model", Dissertation, Universität Bonn, Juni 2006.

[19] D. M. Tolentino Zang, "Final state interactions in strong two-body baryon decays", Dissertation, Universität Bonn, July 2014.

[20] C. Amsler et al. (Particle Data Group), Phys. Lett. B 667, (2008) 1.

[21] T. Melde, W. Plessas, and B. Sengl, Phys. Rev. D 77, 114002 (2008).

[22] M. Ronniger and B. C. Metsch, Eur. Phys. J. A 47 (2011) 162 doi:10.1140/epja/i2011-11162-8 [arXiv:1111.3835 [hep-ph]].

[23] M. Ronniger and B. C. Metsch, Eur. Phys. J. A 49 (2013) 8 doi:10.1140/epja/i2013-13008-9 [arXiv:1207.2640 [hep-ph]].

[24] P. Mergell, U.-G. Meißner and D. Drechsel, Nucl. Phys. A 596, 367 (1996).

[25] M.E. Christy et al., Phys. Rev. C 70, 015206 (2004).

[26] I. A. Qattan et al., Phys. Rev. Lett. 94, 142301 (2005).

[27] A. Bodek, S. Avvakumov, R. Bradford, and H. Budd, J. Phys. Conf. Ser. 110, 082004 (2008).

[28] T. Eden et al., Phys. Rev. C 50, 1749 (1994).

[29] C. Herberg et al., Eur. Phys. J. A 5, 131 (1999).

[30] M. Ostrick et al., Phys. Rev. Lett. 83, 276 (1999).

[31] I. Passchier et al., Phys. Rev. Lett. 82, 4988 (1999).

[32] R. Schiavilla et al., Phys. Rev. C 64, 041002 (2001).

[33] D. Rohe et al., Phys. Rev. Lett. 83, 21 (1999).

[34] J. Golak et al., Phys. Rev. C 63, 034006 (2001). 
[35] H. Zhu et al., Phys. Rev. Lett. 87, 081801 (2001).

[36] R. Madey et al., Phys. Rev. Lett. 91, 122002 (2003).

[37] G. Warren et al., Phys. Rev. Lett. 92, 042301 (2004).

[38] D. I. Glazier et al., Eur. Phys. J. A 24, 101 (2005).

[39] R. Alarcon et al., Eur. Phys. J. A 31, 588 (2007).
[40] T. Bartel et al., Nucl. Phys. B 58, 469 (1973).

[41] H. Anklin et al., Phys. Lett. B 428, 248 (1998).

[42] W. Xu et al., Phys. Rev. Lett. 85 (2000).

[43] G. Kubon et al., Phys. Lett. B 524, 26 (2002). 